\title{
Research on Computer Simulation Modeling Based on Human Movement Mechanics
}

\author{
Feng Zhou \\ School of Physical Education and Health, Hainan Tropical Ocean University, Sanya 572000 , China; \\ zhouff1970@163.com
}

Keywords: Human body dynamics, Modeling, Simulation; DirectX SDK.

\begin{abstract}
In the paper, a form editing and movement simulation control method based on human movement mechanics is presented. DirectX SDK function library is adopted in the method. Reading of human body $3 \mathrm{~d}$ models stored in .X document is realized. Models with hierarchy structure in the standardized part are edited and operated in the aspects of dimension, angle and orientation. Meanwhile, principle of skeletal animation and $4 * 4$ specific transformation matrix analysis are specially proposed in the paper.
\end{abstract}

\section{Introduction}

Research on human body dynamics is a multidisciplinary crossing emerging field. It relates to robot mechanism, kinematics, dynamics, human anatomy, surgery (especially orthopaedics), photogrammetry, testing technology, computer aided design and knowledge in other aspects [1-2]. Human body dynamics modeling and simulation are studied to obtain data about movement and mechanics. It has important significance for guiding design of mechanical and electronic products, sports rehabilitation mechanical design, etc. Human body must be simulated in some experiments as experiment objects in order to meet the needs and security of human beings [3]. Human body should be regarded as abstracts to certain extent in order to ensure human body safety and new equipment research and development. Real condition of human body should be reflected by the extract as far as possible on one hand, and it also should be realized easily on the other hand [4]. Human body model is the foundation of vehicle design, dynamics analysis as well as design and simulation of humanoid robot. Human body model for design belongs to human body geometry/kinematics model. It also provides necessary geometry features for human body dynamics analysis and simulation. Human body model for analysis and simulation belongs to multi-body system dynamics model.

\section{Computer simulation research status quo of human body mechanics}

Research on human body dynamics has long attracted the attention of the people. Italian scientist Leonardo Da Vinci studied various postures and movement of human body as early as the late 15th century. It firstly proposed important idea that 'all living entities capable of movement follow the laws of human body mechanics for movement'. A subject characterized by research on human body mechanics behavior-sports biomechanics was born with rapid development of life science, mechanics and computer technology. Sports biomechanics has been produced and development for less than 60 years in China only as a discipline. Sports biomechanics became active actually until the late 1970s with overall progress of sports science and technology infiltration. It is rapidly developed into one of disciplines which can most embody modern high technology level in sports scientific system [5-6]. Development process of sports biomechanics is investigated and analyzed. Sports biomechanics has made great progress. Meanwhile, we also have to admit that theoretical basis of sports biomechanics is not perfect. Scientific research in the aspect should be pushed forwards continuously.

Human movement analysis modeling is one of forefront directions focused in the field of computer vision in recent years. It is an important technology combining modern biological mechanics and computer vision with very wide and important application field. It is widely applied in the fields of robotics, bionic mechanics, intelligent control, human-computer interaction, movement analysis and virtual reality, etc. Modeling is the core of studying human body movement. Current modeling 
methods include: finite element analysis, multi-rigid-body dynamics, muscle-bone modeling, vibration mechanics, kinematics modeling and experiment methods [7].

\section{Human body movement mechanics modeling and computer simulation principle}

\subsection{Joint movement control principle of human body movement mechanics}

We know that when people raise their arms, the palms connected with the arms are lifted together, and when people raise the upper arms connected with lower arms, the palms and lower arms are raised together according to real human body structure. When people twist necks, heads connected with the necks are rotated for certain angle. When people rotate the spine connected with the necks, the necks and heads are rotated for certain angle. It seems that control acted on one joint is passed to the joints connected with the joint. Meanwhile, joints receiving indirect control also can transfer the control information to next joint connected with it until it reaches the bottom joint [8]. Therefore, it can be seen that human body structure has special and strict connection relationship; the connection relationship affects the body movement mode. Real human body structure is combined. In the paper, schematic diagram of human body model and its connection relationship is shown as figure 1 .

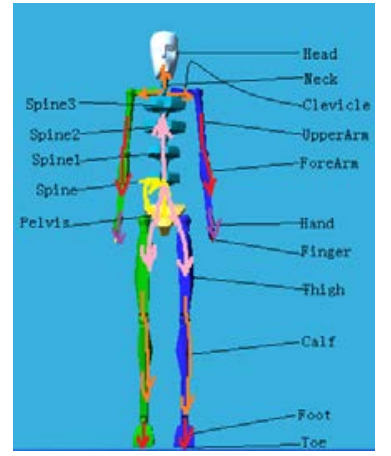

Figure 1 Schematic diagram of 3D human body model structure and connection

Figure 1 shows that arrow direction indicates action transmission direction. It starts from pelvis. It is directly connected with the first spine on the lower portion (it is temporarily called spine 0 ); spinal 0 is connected with spine 1 and left and right thighs. Thighs are connected with legs, soles and toes. Spine 1 is connected with spine 2, spine 3 and neck downwards. Then, the neck is connected with the head and left-right collarbones; collarbones are connected with upper arm, lower arm, palms and fingers.

Common 3D models include standard grid model and skinned mesh model, wherein each joint part is regarded as a unit simply in standard grid model. Corresponding vertex, corresponding material, texture information and a group of transformation matrix are stored. Therefore, influence due to change and transformation of matrix aims at all vertexes of one part in the network model. In addition, the action degree is completely the same. In skinned mesh model, another storage mode is adopted, a series of bones are regulated, and each bone corresponds to a group of specific vertexes in the whole human body model. For example, palm bone corresponds to a series of vertexes on the palm in corresponding model. Similarly, vertexes in skinned network are assigned to different vertex sets according to different belonging bones corresponding to vertex sets in standard network model [9]. It is slightly different in that the vertex in the skinned grid only can absolutely belong to some specific part; thereby it belongs to some specific set. The vertex in the skin network also can belong to many related bones. For example, the vertex on the joint between the upper arm and lower arm belong to two bones at the same time, thereby the vertexes on the joint can be controlled by many transformation matrixes [10].

\subsection{Model sports simulation control based on human body mechanics}

When dynamics model based on human body mechanics is adjusted and control in real time, relative control methods will be more practical. In relative control method, transformation matrix is obtained through original transformation matrix and fine-tuning matrix (hereinafter referred to as fine-tuning matrix) for setting transformation matrix in the frame in the paper. Then, the problem is transfer to how to analyze a combination transformation matrix. 
Vertex transformation matrix proposed in the paper has the following form (the action sequence of agreed rotation transformation is shown as follows: rotation around axis $\mathrm{X}$ - rotation around axis $\mathrm{Y}$ -rotation around axis Z):

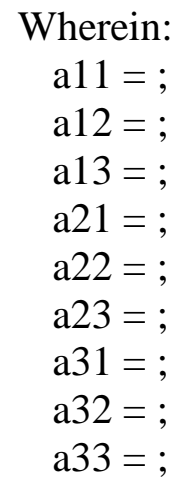

Translation transformation matrix $\mathrm{T}$ can be easily obtained from the combination transformation matrix A. Elements a41, a42 and a43 in matrix A are obtained, which are respectively added into column I, II and III of the first line of $4 * 4$ unit matrix. Elements a41, a42 and a43 in the matrix A are set to be zero for obtaining a combination transformation matrix only containing proportion transformation function and rotation transformation function.

Since control of rotation transformation on the model is conformal, namely it does not change the morphological structure of the controlled object, it just slightly tunes the direction. Proportion transformation matrix has certain influence on morphological structure of the controlled object. It is concretely embodied in changing examples among points in the model. Then, the proportion transformation matrix and rotation transformation matrix can be distinguished from the aspect of difference in the two transformation effects. Four special and extremely representative vertexes are adopted as action objects of transformation from world coordinate system $(1,1,1),(1,1,-1),(1,-1,1)$ and $(-1,1,1)$, and the calculation process is shown in figure 2.

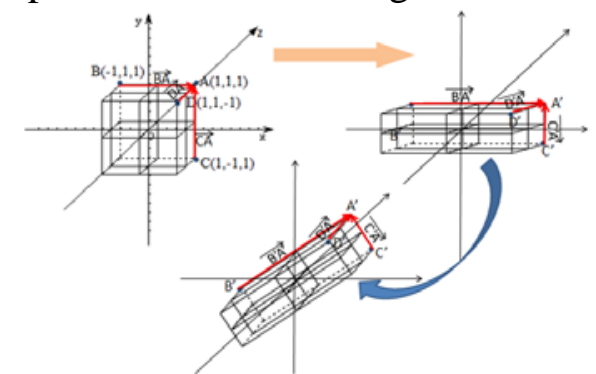

Figure 2 Schematic diagram of proportion transformation matrix parsing process

Figure 2 shows that as long as the coordinates of point $\mathrm{A}, \mathrm{B}, \mathrm{C}$ and $\mathrm{D}$ in the figure after transformation through calculation, new vectors, , are obtained, models of the three vectors can be respectively used for being divided by original vector , , model " 2 ", the scaling of axis $\mathrm{X}$, axis $\mathrm{Y}$ and axis Z Sx, Sy and Sz can be obtained.

Since it is allowed that the scaling on $\mathrm{X}$ axis direction can be negative, the following method is used in the paper for judging whether the scaling on $\mathrm{x}$ direction in actual transformation is negative or not. Obviously, if it is known that obtained in the right upper sub-figure through proportion transformation from is negative to according to figure 2, the scaling on $\mathrm{X}$ axis direction is negative, it is positive if they are on the same direction. However, since the combination transformation matrix of proportion transformation and rotation transformation are acted on the model in one time, they can be directly jumped to the final transformation state from the state before transformation in figure 2, the intermediate state only undergoing proportion transformation can not be obtained. In addition, the figure clearly shows that the vector under the final state is not at the same direction or non-reverse to the vector obviously. It means that it is impossible to assist us to judge the negative and positive signal problem of scaling on $\mathrm{X}$ direction through obtained under final state.

In the paper, characteristics of cross-product show that the breakthrough point to solve problems is obtained. Since the transformation test vertex has certain particularity, namely, the direction met by 
the , , as a result is the same as the direction $\times$. In addition, the proportion scaling on $\mathrm{X}$ axis direction only can affect model and direction. The proportion scaling on Y axis direction only can affect model and direction of . The proportion scaling on $\mathrm{Z}$ axis direction only can affect model and direction of . Since it is agreed that the scaling proportion on $\mathrm{Y}$ axis and $\mathrm{Z}$ axis directions must be greater than 0.000005 , the subsequent rotation transformation can keep relative position relationship of original vertex. It means that if vectors, and are regarded as mutually associated integrity on the direction, we will can obtain direction without scaling on the $\mathrm{X}$ axis direction through calculation through and multiplication cross regardless of positive value scaling on $\mathrm{X}$ axis and $\mathrm{Y}$ axis directions. Since the proportion scaling on $\mathrm{X}$ axis direction only can affect model and direction of , when the scaling on the $\mathrm{X}$ axis direction is positive, the transformation only can change the model size of vector , the direction under the situation is the same as the direction of vector obtained from . When the scaling on the $\mathrm{X}$ axis direction is negative, vector length can be changed by the transformation on one hand, the direction can be negative on the other hand, thereby direction is opposite to the direction of vector obtained from . The above contents are integrated. we can know whether the scaling on the X axis direction is negative or not through judging whether the vector direction of is the same as the vector direction from . The result is positive if they are on the same direction, and negative if they are opposite.

After scaling Sx, Sy ad Sz on X, Y and Z axis directions are parsed, the three values can be obtained for one-time replacement of unit matrix a11, 22 and a33, the proportion transformation matrix contained in the combination transformation matrix can be obtained. The inverse matrix of proportion transformation matrix is multiplied with combination transformation matrix of proportion transformation and rotation transformation on the left, the rotation transformation matrix can be obtained.

\section{Computer simulation modeling realization based on human body mechanics}

\subsection{System overall design}

The computer simulation modeling research system application procedure based on human body dynamics in the paper is compiled by combining Direct3D and MFC application procedure framework. It has the structure relationship shown in figure 3 according to overall framework of the procedure.

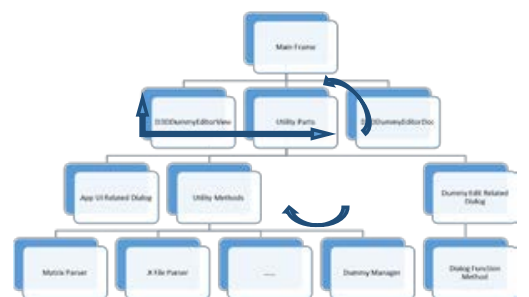

Figure 3 Block diagram of D3DDummyEditor application procedure structure

D3DDummyEditorView class belongs to view class which is obtained with CView class and CXSSDirect3DBasic class as base class, wherein CXSSDirect3DBasic class is self-defined by the author, which contains Direct3D related variables and initialized Direct3D class function. D3DDummyEditorDoc class is a document class derived with CDocument class and CXSSSceneContainer class as base class, wherein CXXSceneContainer class is self-defined by the author, which contains relevant variables and functions for storing and managing models in the scene.

Utility Parts module contains procedure core functions, such as matrix analytic function. X file analytic function and function control realization model, etc. DummyEditRelatedDialog is an application procedure interaction window defined for user control model. The dialogs are mainly based on non-modal dialogs. Corresponding functions in Utility Parts are called for editing and controlling the model according to user indications. When the procedures are operated, the only D3DDummyEditorDoc pointer in the procedure is passed to all model control dialogs. When users select different functions in dialogs, non-modal dialogs can send specific window messages to the view class firstly, and then corresponding processing functions in the dialogs can be activated in the 
view class. The processing functions can directly call the functions in the D3DDummyEditorDoc through the pointer of CD3DDummyEditorDoc. Required functions can be imported to related functions according to variables set by users.

\subsection{System realization}

Application procedure D3DDummyEditor realizes reading of standard grid model and skinned grid model in X file as well as movement simulation control function. In addition, it is allowed that users can store current state for specific model objects, load and configure new morphological characteristics. Since management functions of many models are added in the procedure, users can load in many models at the same time, all models can be edited at the same time or independently. The editing functions on model materials and texture information are also added in the procedure; thereby the model can be controlled from more aspects.

\section{Conclusion}

The above results show that the human body model form editing and sports simulation control method based on human body movement mechanics can realize editing and operation of models with standardized part hierarchy model in the aspects of size, angle and azimuth, thereby realizing modeling computer simulation on human body model. Meanwhile, principle of skeletal animation and $4 \times 4$ specific transformation matrix analysis are specially proposed in the paper.

\section{References}

[1] Jansson J, Vergeest J S M. Combining deformable- and rigid-body mechanics simulation. Visual Computer, 2003, 19(5):280-290.

[2] Lenoir, Julien, Fonteneau, Sylvre. Mixing deformable and rigid-body mechanics simulation. IEEE Computer Society, 2004.

[3] Rasmussen J. Challenges in human body mechanics simulation. Procedia Iutam, 2011, 2(1):176-185.

[4] M. Stelzer, Stryk O V. Efficient forward dynamics simulation and optimization of human body dynamics. Zamm Journal of Applied Mathematics \& Mechanics Zeitschrift Für Angewandte Mathematik Und Mechanik, 2006, 86(10):828-840.

[5] Wang J J. The Visual Simulation Analysis of Human Body Movement Model. Applied Mechanics \& Materials, 2014, 556-562:3913-3916.

[6] Naderi D, Zanoosi A A P, Sadeghi-Mehr M. Forward dynamics simulation of human body under tilting perturbations. Communications in Nonlinear Science \& Numerical Simulation, 2012, 17(2):1055-1064.

[7] Bråse D. A Human Body Model With Active Muscles for Simulation of Pretensioned Restraints in Autonomous Braking Interventions. Traffic Injury Prevention, 2015, 16(3):304-313.

[8] Yeung K W, Li Y, Zhang X. A 3D Biomechanical Human Model for Numerical Simulation of Garment-Body Dynamic Mechanical Interactions During Wear. Journal of the Textile Institute, 2004, 95(1-6):59-79.

[9] Synek, A, Settles, M, Stillfried, G. Multi-body simulation of a human thumb joint by sliding surfaces. 2012:379-384.

[10] Andreescu D, Ionescu F, Riehle H. Modelling and simulation of human body by considering the skeleton's bones. Iasted International Conference on Biomechanics. ACTA Press, 2007:153-160. 